\title{
High mass of the type IIP supernova 2004et inferred from hydrodynamic modeling
}

\author{
V. P. Utrobin ${ }^{1,2}$ and N. N. Chugai ${ }^{3}$ \\ 1 Max-Planck-Institut für Astrophysik, Karl-Schwarzschild-Str. 1, 85741 Garching, Germany \\ 2 Institute of Theoretical and Experimental Physics, B. Cheremushkinskaya St. 25, 117218 Moscow, Russia \\ e-mail: utrobin@itep.ru \\ 3 Institute of Astronomy of Russian Academy of Sciences, Pyatnitskaya St. 48, 109017 Moscow, Russia
}

Received 3 April 2009 / Accepted 7 July 2009

\begin{abstract}
Context. Previous studies of type IIP supernovae have inferred that progenitor masses recovered from hydrodynamic models are higher than $15 M_{\odot}$.

Aims. To verify the progenitor mass of this supernova category, we attempt a parameter determination of the well-observed luminous type IIP supernova 2004et.

Methods. We model the bolometric light curve and the photospheric velocities of SN 2004et by means of hydrodynamic simulations in an extended parameter space.

Results. From hydrodynamic simulations and observational data, we infer a presupernova radius of $1500 \pm 140 R_{\odot}$, an ejecta mass of $24.5 \pm 1 M_{\odot}$, an explosion energy of $(2.3 \pm 0.3) \times 10^{51} \mathrm{erg}$, and a radioactive ${ }^{56} \mathrm{Ni}$ mass of $0.068 \pm 0.009 M_{\odot}$. The estimated progenitor mass on the main sequence is in the range of 25-29 $M_{\odot}$. In addition, we find clear signatures of the explosion asymmetry in the nebular spectra of SN 2004et.

Conclusions. The measured progenitor mass of SN 2004et is significantly higher than the progenitor mass suggested by the preexplosion images. We speculate that the mass inferred from hydrodynamic modeling is overestimated and crucial missing factors are multi-dimensional effects.
\end{abstract}

Key words. stars: supernovae: individual: SN 2004et - stars: supernovae: general

\section{Introduction}

The major parameters of core-collapse supernova $(\mathrm{SN})$ are thought to be linked to the initial stellar mass on the main sequence, the progenitor mass. However, the genealogy of different varieties of $\mathrm{SNe}$ is as yet poorly known. Fortunately, hydrodynamic modeling of the light curves and the expansion velocities allows us to estimate $\mathrm{SN}$ parameters such as a pre-SN radius, an ejecta mass, an explosion energy, and a radioactive ${ }^{56} \mathrm{Ni}$ amount. In the case of SNe IIP, the mass lost prior to the pre-SN stage is relatively small for stars with an initial mass less than $25 M_{\odot}$, so the ejecta mass provides us with a reliable estimate of the progenitor mass or at least its lower limit. Compared to other core-collapse $\mathrm{SNe} \mathrm{Ib} / \mathrm{c}$ and $\mathrm{SNe} \mathrm{IIn}$, we are able to recover the ejecta mass of SNe IIP from hydrodynamic modeling with greater confidence because of both an accurate estimation of the photospheric velocity related to the high opacity of the hydrogen-rich matter, and a low contribution of the circumstellar interaction to the SN luminosity.

Hydrodynamic modeling should only be applied to wellobserved SNe IIP. An adequate simulation of the bolometric light curve and the evolution in the photospheric velocities requires both high-quality photometric and spectroscopic data (Utrobin 2007). For the above reason, hydrodynamic simulations of SNe IIP have been performed for only a handful of events: SN 1987A, SN 1999em, SN 2003Z, and SN 2005cs. For these particular cases, the inferred progenitor masses have been found unexpectedly to be in the range of $15-22 M_{\odot}$ (Utrobin \& Chugai 2008 ), well above the median value of $\approx 13 M_{\odot}$ for the Salpeter initial mass distribution in the mass range of 9-25 $M_{\odot}$ responsible for SNe IIP (Heger et al. 2003), i.e., the progenitor masses are on average more massive than expected.

A more challenging problem is one related to the analysis of the sub-luminous SN 2005cs. The progenitor mass of $\sim 18 M_{\odot}$ inferred from hydrodynamic modeling (Utrobin \& Chugai 2008) was found to significantly exceed the progenitor mass of 6-13 $M_{\odot}$ recovered from archival $H S T$ images (Maund et al. 2005; Li et al. 2006; Eldridge et al. 2007). No reasonable explanation of this disparity has been proposed.

To pinpoint the cause of this disagreement between the two methods of the mass determination, one needs to confirm this discrepancy in mass measurements for a larger sample of SNe IIP with a broad range of observational characteristics. In this respect, the well-observed luminous SN 2004et in the nearby galaxy NGC 6946 is a particularly favorable case. This object discovered soon after its explosion has the highest intrinsic luminosity among well-studied events (Sahu et al. 2006) and, perhaps, the highest ejecta mass and explosion energy. In this case, the progenitor was directly identified in the archival images $(\mathrm{Li}$ et al. 2005).

Here we perform hydrodynamic modeling of SN 2004et to recover the basic parameters: pre-SN radius, ejecta mass, explosion energy, and radioactive ${ }^{56} \mathrm{Ni}$ mass. A brief description of the hydrodynamic model is given in Sect. 2.1, and the basic parameters of the optimal model are obtained in Sect. 2.2. In Sect. 2.3, we investigate whether the non-evolutionary model should be used instead of evolutionary pre-SN for the 
one-dimensional hydrodynamic modeling of SN 2004et and in general SNe IIP. The progenitor mass of SN 2004et is evaluated and compared to estimations for other SNe IIP (Sect. 2.4). The measured progenitor mass noticeably exceeds the mass estimated from the pre-explosion images, and this disagreement is discussed in Sect. 3.1. In particular, we propose that the explosion asymmetry could be responsible for the disagreement in mass estimates and explore signatures of the explosion asymmetry in the SN 2004et nebular spectra (Sect. 3.2). Finally, in Sect. 4, we summarize the results obtained.

We adopt a distance to NGC 6946 of $5.5 \mathrm{Mpc}$ and a reddening $E(B-V)=0.41$ as measured by Li et al. (2005), an explosion date on September 22.0 UT (JD 2453 270.5), and a recession velocity to the host galaxy of $45 \mathrm{~km} \mathrm{~s}^{-1}$ following Sahu et al. (2006).

\section{Hydrodynamic model and progenitor mass}

\subsection{Model overview}

The modeling of the SN explosion is performed using the spherically-symmetric hydrodynamic code with one-group radiation transfer (Utrobin 2004, 2007), which has been applied previously to other SNe IIP. Utrobin (2007) found that both this one-group approach and the multi-group approach of Baklanov et al. (2005) measured similar ejecta mass and explosion energy for SN 1999em. The basic equations and details of the input physics, including calculations of mean opacities, are described in Utrobin (2004). The present version of the code includes additionally Compton cooling and heating. The explosion energy is modeled by placing the supersonic piston close to the outer edge of the $1.6 M_{\odot}$ central core, which is removed from the computational mass domain and assumed to collapse to become a neutron star. The principal limitation of the code is that the explosion asymmetry and the Rayleigh-Taylor mixing between the helium core and hydrogen envelope (Müller et al. 1991) cannot be correctly treated by the one-dimensional model. We, therefore, study a "non-evolutionary" pre-SN, which takes into account the result of the mixing during the explosion and the shock propagation in the evolutionary pre-SN. The distinctive feature of the non-evolutionary model is a smoothed density and composition jumps between the helium core and the hydrogen envelope.

The resultant structure of the non-evolutionary pre-SN in our optimal model is shown in Fig. 1. The pre-SN model is defined to be a red supergiant (RSG) with a radius of $1500 R_{\odot}$, three times larger than in the case of the normal type IIP SN 1999em (Utrobin 2007). This large pre-SN radius for SN 2004et is implied by the broad initial peak of the bolometric light curve shown by Sahu et al. (2006). The adopted mixing between the helium core and hydrogen envelope in the optimal model is shown in Fig. 2. The degree of mixing determines the light curve at the end of the plateau (Utrobin et al. 2007). The unmixed helium-core mass adopted for SN 2004et is $8.1 M_{\odot}$, which corresponds to the final helium core of a main-sequence star of $\approx 25 M_{\odot}$ (Hirschi et al. 2004). We note that the model light curve is not sensitive to any variation in the helium-core mass of the mixed model (Utrobin et al. 2007).

\subsection{Basic parameters}

The search for the best-fit model parameters is performed by computations of an extensive grid of hydrodynamic models. The optimal model should reproduce simultaneously the bolometric
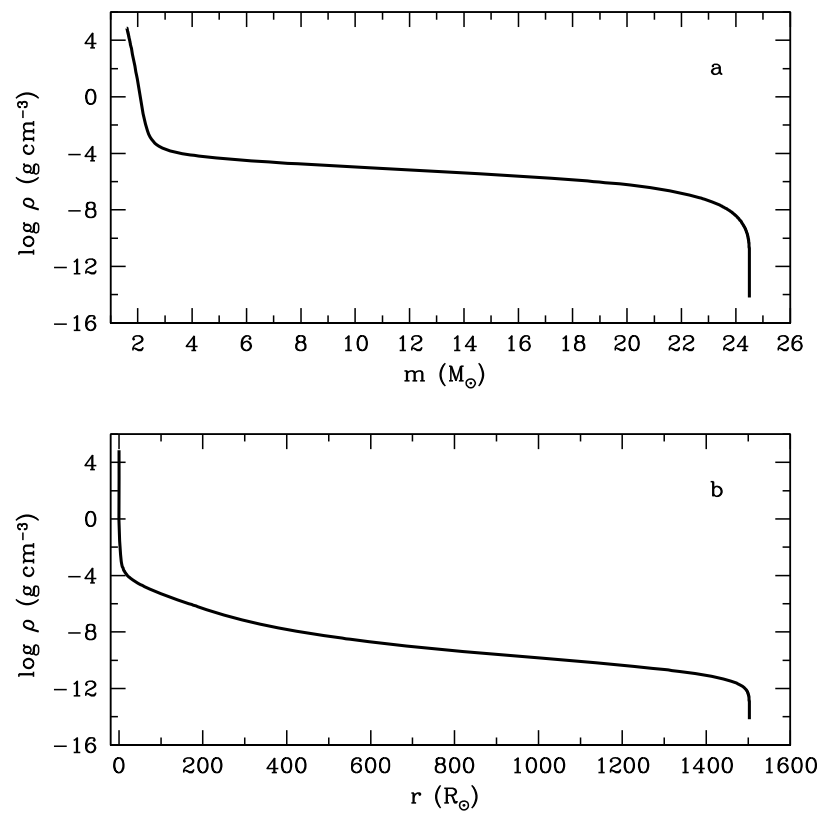

Fig. 1. Density distribution as a function of interior mass a) and radius b) for the optimal pre-SN model. The central core of $1.6 M_{\odot}$ is omitted.

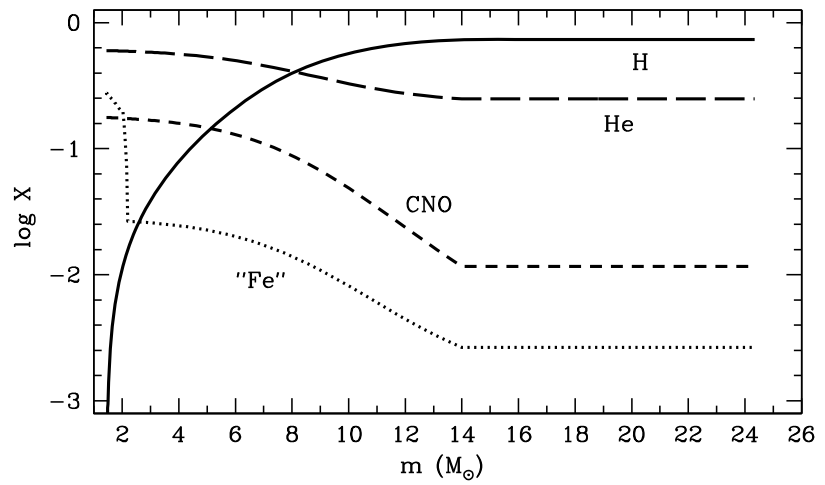

Fig. 2. The mass fraction of hydrogen (solid line), helium (long dashed line), CNO elements (short dashed line), and Fe-peak elements including radioactive ${ }^{56} \mathrm{Ni}$ (dotted line) in the ejecta of the optimal model.

light curve and the photospheric velocity evolution in the best way. As a result, we derive the ejecta mass $M_{\text {env }}=22.9 \pm 1 M_{\odot}$, the explosion energy $E=(2.3 \pm 0.3) \times 10^{51} \mathrm{erg}$, the pre-SN radius $R_{0}=1500 \pm 140 R_{\odot}$, and the ${ }^{56} \mathrm{Ni}$ mass $M_{\mathrm{Ni}}=0.068 \pm$ $0.009 M_{\odot}$. The uncertainties in the basic parameters are calculated by assuming relative errors in the input observational data: $11 \%$ in the distance, $7 \%$ in the dust absorption, $5 \%$ in the photospheric velocity, and $2 \%$ in the plateau duration. In general, the errors of derived parameters should be somewhat larger because of model systematic errors. However, the latter cannot be confidently estimated unless a more advanced and correct model is developed. The model uncertainties will be discussed below in Sect. 3.2.

The density distribution in the freely expanding SN envelope is shown in Fig. 3. Multiple shells in the outermost layers with velocities $v>14000 \mathrm{~km} \mathrm{~s}^{-1}$ (Fig. 3) form at the shock breakout stage by the radiative acceleration in the optically thin regime. The origin of these shells is related to the specific behavior of the line opacity in the outer rarefied layers of temperature $\sim 10^{5} \mathrm{~K}$. The innermost shell of mass $\sim 5 \times 10^{-3} M_{\odot}$ at the velocity of $11700 \mathrm{~km} \mathrm{~s}^{-1}$ is the thin shell formed by the effect of the shock 


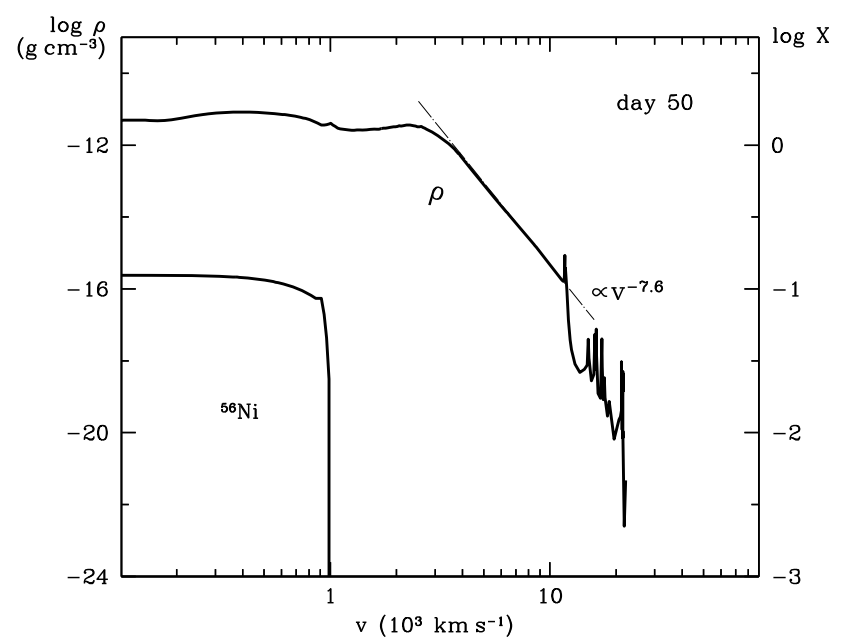

Fig. 3. The density and the ${ }^{56} \mathrm{Ni}$ mass fraction as a function of velocity for the optimal model at $t=50$ days. Dash-dotted line is the density distribution fit $\rho \propto v^{-7.6}$.

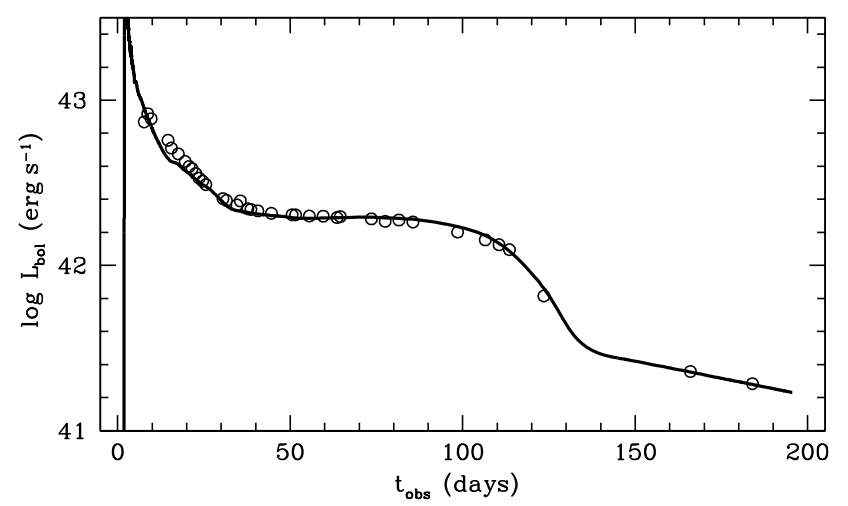

Fig. 4. The calculated bolometric light curve of the optimal model (solid line) overplotted on the bolometric data of SN 2004et evaluated from the UBVRI observations of Sahu et al. (2006) (open circles).

breakout in the optically thick regime (Grassberg et al. 1971; Chevalier 1981).

The optimal model describes the bolometric light curve quite well, including its initial $(t<30$ days) peak (Fig. 4). This peak is substantially broader and more luminous than the initial peak of SN 1999em (cf. Sahu et al. 2006). It is the initial luminosity peak of SN 2004et that requires the larger radius of the pre-SN model compared to the pre-SN radius of $500 R_{\odot}$ in the case of SN 1999em (Utrobin 2007).

With one-group radiation transfer, the hydrodynamic model is not assumed to reproduce the monochromatic light curves in detail. However, it is instructive to compare the model and the observations in $B, V$, and $R$ bands (Figs. $5 \mathrm{a}-\mathrm{c}$ ). The $B$ light curve is reproduced at the initial hot phase, but not at the late phase. The overall fit of the $V$ light curve is much tighter. In $R$ band, the calculated light curve reproduces the plateau data, but does not describe the very initial stages of the light curve. The differences between the hydrodynamic model and the observations are related to deviations of the $\mathrm{SN}$ spectrum from a blackbody. These deviations are significant in the blue and ultraviolet at late photospheric epochs, which explains why the disagreement is strongest in $B$ band.

The computed photospheric velocity is shown together with two sets of observational data (Fig. 5d): the first is recovered from an absorption minimum of the Fe II $5169 \AA$ line
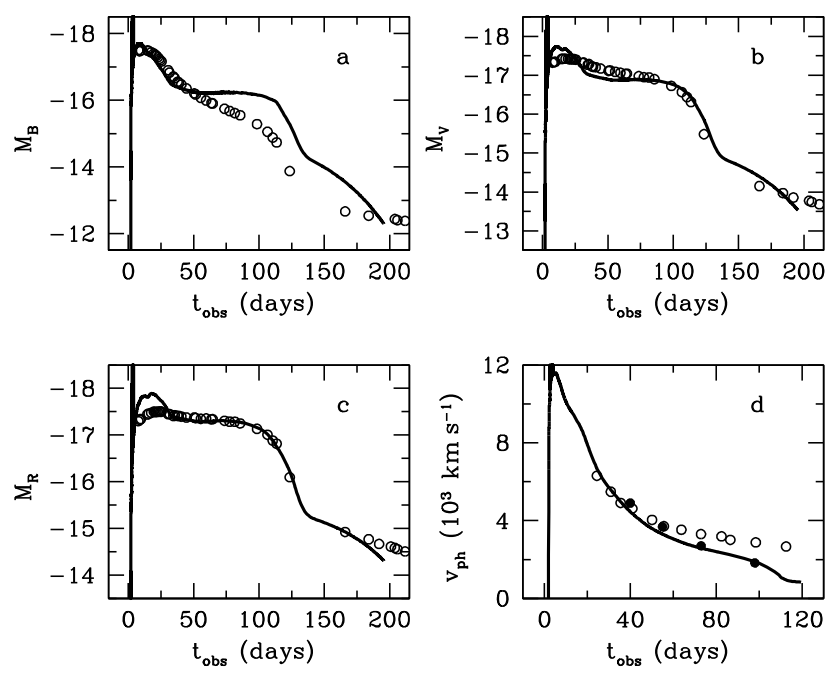

Fig. 5. Optimal hydrodynamic model. Panels a)-c): the calculated $B$, $V$, and $R$ light curves (solid lines) compared with the corresponding observations of SN 2004et obtained by Sahu et al. (2006) (open circles). Panel d): the calculated photospheric velocity (solid line) is compared with photospheric velocities estimated from the absorption minimum of the Fe II 5169 A line (open circles) by Sahu et al. (2006) and recovered from the Na I doublet profile (filled circles).

(Sahu et al. 2006) and the second, from our modeling of the $\mathrm{Na}$ I doublet profile. The latter photospheric velocities can be measured more confidently than those recovered from absorption minima, especially at late photospheric epochs when absorption lines become strong. The model photospheric velocity is consistent with both the Na I data and the early data of the Fe II $5169 \AA$ absorption minimum. We also modeled profiles of the Fe II 4924, 5018, $5169 \AA$ A lines at late photospheric stages, and found that the photospheric velocities obtained from these lines were rather similar to velocities obtained by modeling the Na I doublet profile. Unfortunately, the spectral data of SN 2004et for the early stages are missing. The blue edge of the $\mathrm{H} \alpha$ absorption in the earliest spectrum on day 24 implies a maximal expansion velocity of $\sim 12000-13000 \mathrm{~km} \mathrm{~s}^{-1}$ in the ejecta. This velocity is consistent with the model maximal velocity of $12000 \mathrm{~km} \mathrm{~s}^{-1}$.

\subsection{Explosion of evolutionary presupernova}

The arguments in favor of the non-evolutionary pre-SN model leave some doubts and raise a question: why should we not consider an evolutionary pre-SN? This issue has already been explored for the sub-luminous type IIP SN 2005cs (Utrobin \& Chugai 2008), for which we found that the evolutionary preSN did not allow us to produce a realistic description of both the light curve and the photospheric velocities. A similar problem was encountered while modeling the explosion of evolutionary pre-SNe with other hydrodynamic codes (Chieffi et al. 2003; Woosley \& Heger 2007).

We therefore check whether the same problem holds for SN 2004et, which differs in terms of both ejecta mass and explosion energy from SN $2005 \mathrm{cs}$. We adopt the pre-SN model with an envelope mass of $15.9 M_{\odot}$ and a density distribution that closely resembles that of the evolutionary model (Utrobin \& Chugai 2008). The hydrogen and helium are assumed to be mixed along the mass coordinate in a similar way to the optimal model (Fig. 2). An optimal fit to the bolometric light curve and 

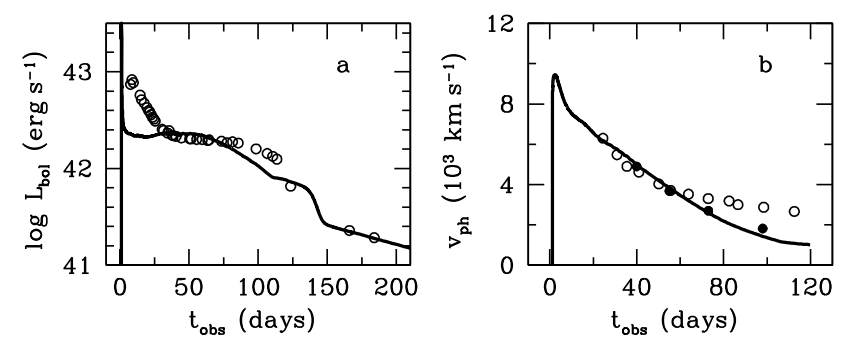

Fig. 6. Hydrodynamic model of SN 2004et for the evolutionary pre-SN. Panel a): the model bolometric light curve (solid line) overplotted on the observational data (see Fig. 4 legend for details). Panel b): the model photospheric velocity (solid line) and the observational photospheric velocities (see Fig. 5d legend for details).

the evolution in photospheric velocity is attained for an explosion energy of $1.3 \times 10^{51} \mathrm{erg}$ and a pre-SN radius of $600 R_{\odot}$. Apparent disadvantages of the obtained model are an extremely narrow initial peak of luminosity and a two step-like transition from the plateau to the radioactive tail (Fig. 6a). The latter behavior is similar to that demonstrated by the light curves computed for the evolutionary pre-SN by Woosley \& Heger (2007). The narrow initial peak is related to the relatively small pre-SN radius. However, one cannot increase the pre-SN radius to obtain a superior fit because the photospheric velocity would then become unacceptably low. A larger initial radius would produce a higher luminosity, which, in turn, should be compensated by a decrease in the explosion energy, consequently, leading to lower expansion velocities. Even in the demonstrated model, the maximal velocity is only $9500 \mathrm{~km} \mathrm{~s}^{-1}$ (Fig. 6b), significantly lower than the observed maximal velocity of $12000-13000 \mathrm{~km} \mathrm{~s}^{-1}$. We also computed the same model but without mixing between the helium core and the hydrogen-rich envelope. This model provides an even poorer fit because the "bump" at the end of the plateau becomes more boxy, in sharp contrast to the observational light curve.

To summarize, the model including an explosion of the evolutionary pre-SN does not allow us to achieve a close fit to the bolometric light curve and the maximal expansion velocities of SN 2004et. This problem is not related to the evolutionary pre-SN itself. The one-dimensional hydrodynamics cannot reproduce the outcome of a real explosion in the evolutionary model, because multi-dimensional effects, in particular mixing between the helium core and the hydrogen envelope, play a crucial role during the explosion and shock propagation phases. The two-dimensional hydrodynamic model predicts that the Rayleigh-Taylor mixing at the helium/hydrogen interface reduces the high composition and density gradients (Müller et al. 1991). Our non-evolutionary pre-SN qualitatively takes this multi-dimensional effect into account, which ensures that the non-evolutionary model describes the light curve shape more successfully. A non-evolutionary pre-SN is also preferred by Baklanov et al. (2005) in their modeling of SN 1999em.

\subsection{Progenitor mass}

The ejecta mass $M_{\mathrm{env}}=22.9 \pm 1 M_{\odot}$ combined with the collapsing core of $1.6 M_{\odot}$ yields the pre-SN mass of $24.5 \pm 1 M_{\odot}$. A progenitor mass on the main sequence is higher by the amount of matter lost via the wind at the main-sequence and RSG phases. For the main-sequence stage, we rely on the computations of Meynet \& Maeder (2003) for non-rotating stars with the massloss rate of Vink et al. (2001). They found that a star with an
Table 1. Hydrodynamic models of type IIP supernovae.

\begin{tabular}{lcccccc}
\hline \hline SN & $\begin{array}{c}R_{0} \\
\left(R_{\odot}\right)\end{array}$ & $\begin{array}{c}M_{\text {env }} \\
\left(M_{\odot}\right)\end{array}$ & $\begin{array}{c}E \\
\left(10^{51} \mathrm{erg}\right)\end{array}$ & $\begin{array}{c}M_{\mathrm{Ni}} \\
\left(10^{-2} M_{\odot}\right)\end{array}$ & $\begin{array}{c}v_{\mathrm{Ni}}^{\max } \\
\left(\mathrm{km} \mathrm{s}^{-1}\right)\end{array}$ \\
\hline $1987 \mathrm{~A}$ & 35 & 18 & 1.5 & 7.65 & 3000 & 600 \\
$1999 \mathrm{em}$ & 500 & 19 & 1.3 & 3.60 & 660 & 700 \\
$2003 Z$ & 229 & 14 & 0.245 & 0.63 & 535 & 360 \\
$2004 \mathrm{et}$ & 1500 & 22.9 & 2.3 & 6.8 & 1000 & 300 \\
$2005 \mathrm{cs}$ & 600 & 15.9 & 0.41 & 0.82 & 610 & 300 \\
\hline
\end{tabular}

initial mass $M_{\text {ZAMS }}=25 M_{\odot}$ lost $0.8 M_{\odot}$ during the main sequence (Meynet \& Maeder 2003). For the RSG stage, Meynet $\&$ Maeder (2003) used the mass-loss rate of de Jager (1988) and predicted that $25 M_{\odot}$ and $40 M_{\odot}$ main-sequence stars lost $7.5 M_{\odot}$ and $21 M_{\odot}$, respectively. With these estimates of the lost mass, we come to the progenitor mass of SN 2004et in the range of $30-40 M_{\odot}$.

A less massive progenitor is expected if we use the wind density recovered for the SN 2004et pre-SN from X-ray observations. These data suggest the mass-loss rate of $(2-2.5) \times$ $10^{-6} M_{\odot} \mathrm{yr}^{-1}$, assuming the wind velocity of $10 \mathrm{~km} \mathrm{~s}^{-1}$ (Rho et al. 2007; Misra et al. 2007). Using the RSG life-time of $7 \times$ $10^{5} \mathrm{yr}$ for the $25 M_{\odot}$ main-sequence star (Hirshi et al. 2004), we find the mass lost at the RSG phase to be $\sim 1.6 M_{\odot}$ with an uncertainty of $\sim \pm 1 M_{\odot}$. With the mass of $0.8 M_{\odot}$ lost at the main-sequence phase (Hirshi et al. 2004), the total lost mass is then $2.4 \pm 1 M_{\odot}$. The pre-SN mass of $24.5 M_{\odot}$ combined with the lost mass results in the progenitor mass of $27 \pm 2 M_{\odot}$, where the error includes the uncertainties in the ejecta mass and the mass-loss rate. The progenitor mass of SN 2004et turns out to be close to the maximal initial mass for SNe IIP according to the present-day paradigm (Heger et al. 2003).

Table 1 presents the parameters of all the SNe IIP studied hydrodynamically. The listed parameters are the pre-SN radius, the ejecta mass, the explosion energy, the total ${ }^{56} \mathrm{Ni}$ mass, the maximal velocity of ${ }^{56} \mathrm{Ni}$ mixing zone, and the minimal velocity of the hydrogen-rich envelope. The ejecta and progenitor masses of SN 2004et are found to be maximal among the well-studied SNe IIP. With the exception of the initial radius for SN 1987A, the explosion energy and the total ${ }^{56} \mathrm{Ni}$ mass show the most extreme variations (of one order of magnitude). All SNe IIP are characterized by a deep mixing of hydrogen, indicated by the low value of $v_{\mathrm{H}}^{\mathrm{min}}$, which is consistent with two-dimensional simulations (Müller et al. 1991; Kifonidis et al. 2003, 2006). The position of SN 2004et on the plots of explosion energy versus progenitor mass (Fig. 7a) and the total ${ }^{56} \mathrm{Ni}$ mass versus the progenitor mass (Fig. 7b) strengthens the correlations recovered earlier for the SNe IIP studied hydrodynamically (Utrobin \& Chugai 2008). We note that these correlations also infer the correlation between the explosion energy and the total ${ }^{56} \mathrm{Ni}$ mass, which was found and discussed earlier by Nadyozhin (2003).

\section{Discussion}

\subsection{Whether the hydrodynamic mass is overestimated?}

The pre-SN mass recovered from the hydrodynamic modeling is very much close to the progenitor mass. It is, therefore, reasonable to refer to the progenitor mass thus determined as the "hydrodynamic" mass. Surprisingly, the hydrodynamic mass of the SN 2004et progenitor, $27 \pm 2 M_{\odot}$, noticeably exceeds, by a factor of $\sim 2-3$, the value of $9_{-1}^{+5} M_{\odot}$ recovered from the analysis of pre-explosion images (Smartt et al. 2009). A similarly large 


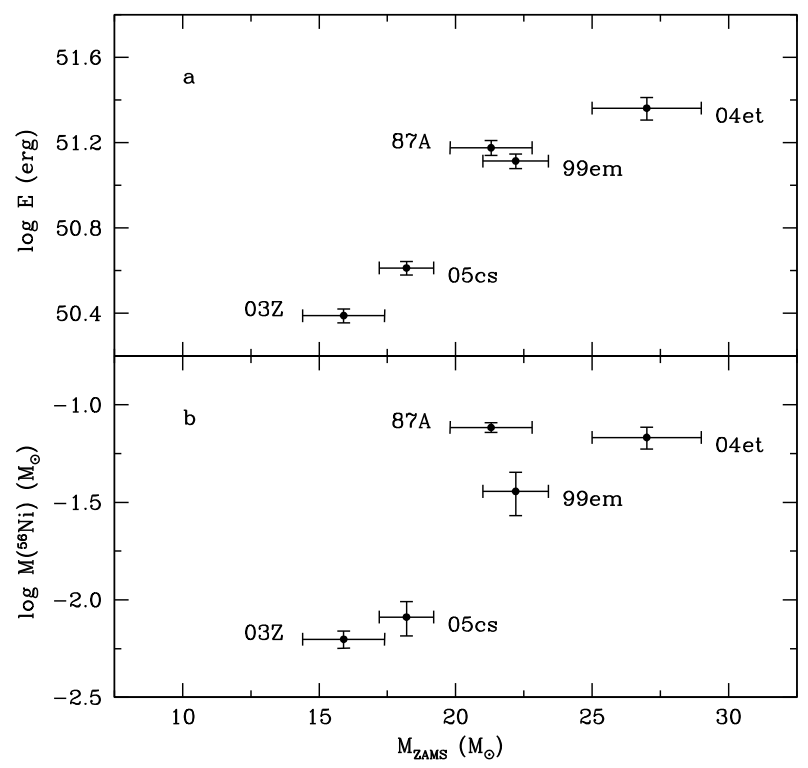

Fig. 7. Explosion energy a) and ${ }^{56} \mathrm{Ni}$ mass b) versus hydrodynamic progenitor mass for five core-collapse $\mathrm{SNe}$.

mass disparity has been found for SN 2005cs: $18 M_{\odot}$ (Utrobin \& Chugai 2008) versus 6-13 $M_{\odot}$ (Maund et al. 2005; Li et al. 2006; Eldridge et al. 2007). These two cases of huge discrepancies in progenitor mass clearly illustrate the high level of uncertainty in the mass problem.

The other side of this problem is that all the known hydrodynamic masses of SNe IIP progenitors are in the range $M_{\text {ZAMS }}>15 M_{\odot}$ (Fig. 7). This mass distribution conflicts with the paradigm that SNe IIP originate from the mass range of 9-25 $M_{\odot}$ (Heger et al. 2003). Assuming the Salpeter initial mass function for $\mathrm{SNe}$ IIP rate in the 9-25 $M_{\odot}$ mass range and neglecting selection effects, we expect that five $\mathrm{SNe}$ should be found in the 15-25 $M_{\odot}$ mass range with a probability of only 0.004 .

A quite different conclusion was reached by Smartt et al. (2009). They conclude that SNe IIP progenitors detected or undetected in pre-explosion images have masses in the range of 8-17 $M_{\odot}$. This significant difference in the progenitor-mass distributions obtained by two methods and the mass discrepancy for SN 2004et and SN 2005cs again implies that the determination of the progenitor mass remains a difficult problem. Although the low masses of SNe IIP progenitors from archival images also pose a problem for the fate of massive RSG stars in the range of 17-25 $M_{\odot}$ (Smartt et al. 2009), we address here only the possibility that hydrodynamic masses are strongly overestimated compared to the real progenitor masses.

\subsection{Could explosion asymmetry be a crucial missing factor?}

To address the disagreements between progenitor mass determinations, we should consider possible problems with our numerical modeling. Among the missing factors that might affect the accuracy of the inferred SN parameters, the most apparent are: multi-group radiation transfer, full non-LTE treatment of gas excitation, time-dependent ionization, high spacial resolution of the shock front, multi-dimensional effects of the explosion and shock propagation, consideration of density perturbations related to vigorous convection in the RSG envelope. The effects produced by these factors perhaps differ in magnitude and some of the factors could be insignificant. However,
Table 2. Components of oxygen line decomposition.

\begin{tabular}{lccccc}
\hline \hline Component & $\begin{array}{c}v \\
\left(\mathrm{~km} \mathrm{~s}^{-1}\right)\end{array}$ & $A_{1}$ & $A_{2}$ & $\begin{array}{c}n \\
\mathrm{~cm}^{-3}\end{array}$ \\
\hline symmetric & 0 & 1800 & 0.59 & 0.27 & $1.25 \times 10^{9}$ \\
red & 1080 & 900 & 0.14 & 0.049 & $2.0 \times 10^{8}$ \\
blue & -450 & 480 & 0.32 & 0.20 & $2.8 \times 10^{9}$ \\
\hline
\end{tabular}

detailed numerical studies based on advanced hydrodynamic models are needed to estimate the role of each factor. At present, we can only state firmly that the multi-group treatment of radiation transfer cannot notably change the inferred $\mathrm{SN}$ parameters. This conclusion is based on the comparison between the multigroup (Baklanov et al. 2005) and one-group (Utrobin 2007) approaches to the SN 1999em modeling.

A major drawback of our model may be its one-dimensional approximation. The multi-dimensional effects related to the Rayleigh-Taylor mixing between the helium core and the hydrogen envelope during the shock propagation smear the composition and density jumps (Müller et al. 1991). These effects are included artificially into our pre-SN model. More careful treatment of these effects with multi-dimensional radiation hydrodynamics could modify the inferred SN parameters.

Another multi-dimensional effect, which could potentially be of importance, is the explosion asphericity. A growing amount of observational data favor a picture in which the explosion of SNe IIP is initiated by bipolar jets. SN 1987A provided us with the first evidence of explosion asymmetry inferred from polarization (cf. Jeffery 1991), line asymmetry (Haas et al. 1990), and direct imaging (Wang et al. 2002). During the last decade polarization has been detected in another five SNe IIP (Leonard \& Filippenko 2001; Leonard et al. 2001, 2006). Two of these SNe IIP, SN 1999em and SN 2004dj, exhibit pronounced asymmetry in their $\mathrm{H} \alpha$ emission at the nebular stage, which is interpreted to be caused by asymmetric jets of ${ }^{56} \mathrm{Ni}$ (Chugai 2007). The absence of a pronounced polarization at the early photospheric epoch indicates that the explosion asymmetry does not lead to the asphericity of the hydrogen envelope (Leonard et al. 2001, 2006). The spherization would develop more successfully, if bipolar jets are thermal energy dominated (Couch et al. 2009). However, the spherization of jets in the hydrogen envelope does not preclude that the asymmetric explosion could result in the modification of the velocity-mass distribution compared to the spherical explosion. The disagreement between the mass measurements may then be resolved, if the asymmetric explosion reproduces the observed light curve and expansion velocities for the essentially lower ejecta mass compared to the one-dimensional explosion model. To verify this possibility, we would require multi-dimensional hydrodynamic modeling.

Using the spectra obtained by Sahu et al. (2006), we checked whether SN 2004et exhibited signatures of the explosion asymmetry. We found that nebular H $\alpha$ and [O I] 6300, $6364 \AA$ lines indeed exhibit asymmetry. To quantify asymmetry effects, each line of the oxygen doublet on day 301 was decomposed into three Gaussian components: symmetric, red, and blue. The intensities of the corresponding components in blue and red lines of the doublet were free parameters. Because the line optical depth affects the doublet ratio, as in SN 1987A (Chugai 1988), the line ratio permits us to recover the Sobolev optical depth and, therefore, the oxygen number density. The effect is weakly dependent on the electron temperature, which is assumed to be $5000 \mathrm{~K}$. 


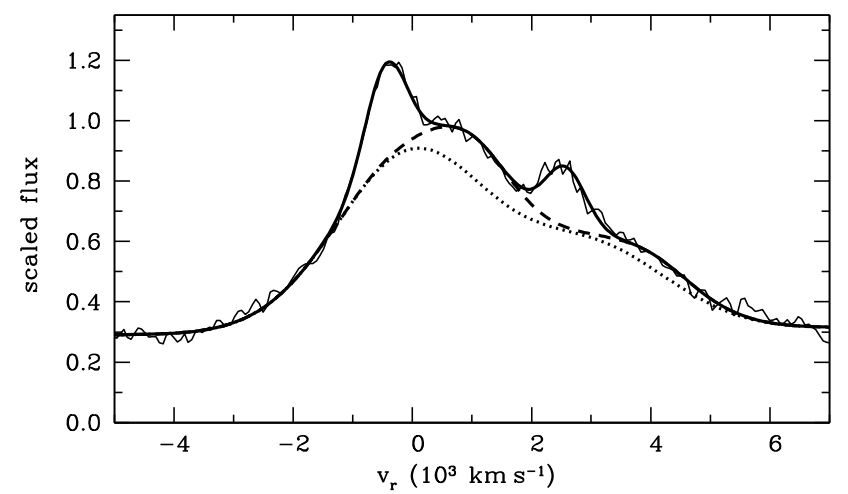

Fig. 8. Oxygen doublet [O I] 6300, $6364 \AA$ observed on day 301 (thin solid line). Zero radial velocity corresponds to the rest wavelength of $6300 \AA$ A Thick solid line is the model doublet profile with all three Gaussian components, dashed line is the profile without the blue component, and dotted line is the symmetric component.

The result of the doublet synthesis is shown in Fig. 8 with the model parameters listed in Table 2. The table columns give the radial velocity shift of the Gaussian component $(v)$, its Doppler width $(u)$, the amplitudes of its blue $\left(A_{1}\right)$ and red $\left(A_{2}\right)$ doublet components, and the number density of the line-emitting oxygen determined from the doublet ratio of each Gaussian component. We emphasize that the decomposition is not unique unless we constrain ourselves by the shape and number of components. The adopted decomposition procedure leads to a minimal contribution of the asymmetric components. Although the red and blue components are weaker than the symmetric one, the asymmetry is rather pronounced. Both asymmetric components have comparable integrated fluxes, but are not identical in terms of the Doppler widths and velocity shifts. These results infer a bipolar structure and a deviation from the point symmetry of the lineemitting gas in the inner layers of the SN envelope. We note our conclusion refers to the line-emitting gas, which is not identical to the overall oxygen distribution. It may well be that the asymmetry of the line-emitting oxygen is related primarily to the asymmetry of ${ }^{56} \mathrm{Ni}$ ejecta (Chugai 2007). Interestingly, a combination of the bipolar structure and the deviation from the point symmetry is a specific feature of SN 1987A, SN 1999em, and SN 2004dj (Chugai 2007). The case of SN 2004et thus provides further support to the conjecture that the explosion asymmetry is an ubiquitous phenomenon of SNe IIP.

Remarkably, the oxygen number density for the symmetric and blue components (Table 2) is comparable, to within a factor of unity, with the oxygen density of $(0.5-1.4) \times 10^{9} \mathrm{~cm}^{-3}$ for the optimal model in the velocity range $v<2500 \mathrm{~km} \mathrm{~s}^{-1}$. In the latter case, we assume that the oxygen density is equal to the total matter density. This coincidence suggests that whatever the role of asymmetry, the related effects do not strongly modify the density distribution of our optimal model in the inner layers with velocities $v<2000 \mathrm{~km} \mathrm{~s}^{-1}$. Of course, this does not preclude that the velocity-density distribution in outer layers could be modified significantly as a result of the aspherical explosion.

\section{Conclusions}

Our goal was to recover the parameters of the hydrodynamic model of the luminous type IIP SN 2004et. We obtained the optimal parameter set: the ejecta mass $M_{\mathrm{env}}=22.9 \pm 1 M_{\odot}$, the explosion energy $E=(2.3 \pm 0.3) \times 10^{51} \mathrm{erg}$, the pre-SN radius $R_{0}=1500 \pm 140 R_{\odot}$, and the ${ }^{56} \mathrm{Ni}$ mass $M_{\mathrm{Ni}}=0.068 \pm$ $0.009 M_{\odot}$. The inferred ejecta mass and explosion energy are maximal among all the known SNe IIP explored by means of radiation hydrodynamics. The parameters of SN 2004et strengthen correlations between the explosion energy and progenitor mass, and between the total ${ }^{56} \mathrm{Ni}$ mass and progenitor mass discussed earlier (Utrobin \& Chugai 2008).

The progenitor mass of SN 2004et, estimated by combining the pre-SN mass and the mass lost via the stellar wind, turns out to be significantly, by a factor of $2-3$, higher than the mainsequence mass recovered from the pre-explosion images. This and the disagreement between mass estimates found earlier for SN 2005cs raise serious concern about the reliability of the progenitor mass recovered from the hydrodynamic modeling. We speculate that among the pitfalls of our hydrodynamic code, the most crucial could be the one-dimensional approximation. The artificial mixing between the helium core and the hydrogen envelope, which we use to simulate real mixing, could be flawed, while explosion asphericity is completely ignored. The evidence of the explosion asphericity of SN 2004et is inferred from the nebular lines, which thus supports the view that the explosion asymmetry is an ubiquitous phenomenon for SNe IIP.

Acknowledgements. We are indebted to D. K. Sahu for sending us spectra of SN 2004et. One of us (VU) is grateful to Wolfgang Hillebrandt for the possibility to work at the MPA. We thank the anonymous referee for useful comments on our manuscript.

\section{References}

Baklanov, P. V., Blinnikov, S. I., \& Pavlyuk, N. N. 2005, Astron. Lett., 31, 429 Chevalier, R. A. 1981, Fundam. Cosmic Phys., 7, 1

Chieffi, A., Domínguez, I., Höflich, P., Limongi, M., \& Straniero, O. 2003, MNRAS, 345, 111

Chugai, N. N. 1988, ATsir, 1525, 15

Chugai, N. N. 2007, in Supernova 1987A: 20 years after. Supernovae and Gamma-ray Bursters, ed. S. Immler, K. Weiler, \& R. McCray, AIP Conf. Proc., 937, 357

Couch, S. M., Wheeler, J. C., \& Milosavljevic, M. 2009, ApJ, 696, 953

de Jager, C., Nieuwenhuijzen, H., \& van der Hucht, K. A. 1988, A\&AS, 72, 259 Eldridge, J. J., Mattila, S., \& Smartt, S. J. 2007, MNRAS, 376, L52

Grassberg, E. K., Imshennik, V. S., \& Nadyozhin, D. K. 1971, Ap\&SS, 10, 28

Haas, M. R., Colgan, S. W. J., Erickson, E. F., et al. 1990, ApJ, 360, 257

Heger, A., Fryer, C. L., Woosley, S. E., Langer, N., \& Hartmann, D. H. 2003, ApJ, 591, 288

Hirschi, R., Meynet, G., \& Maeder, A. 2004, A\&A, 425, 649

Jeffery, D. J. 1991, ApJS, 77, 405

Kifonidis, K., Plewa, T., Scheck, L., Janka, H.-Th., \& Müller, E. 2003, A\&A, 408,621

Kifonidis, K., Plewa, T., Scheck, L., Janka, H.-Th., \& Müller, E. 2006, A\&A, 453,661

Leonard, D. C., \& Filippenko, A. V. 2001, PASP, 113, 920

Leonard, D. C., Filippenko, A. V., \& Ardila, D. R. 2001, ApJ, 553, 861

Leonard, D. C., Filippenko, A. V., Ganeshalingam, M., et al. 2006, Nature, 440, 505

Li, W., Van Dyk, S. D., Filippenko, A. V., \& Cuillandre, J.-C. 2005, PASP, 117, 121

Li, W., Van Dyk, S. D., Filippenko, A. V., et al. 2006, ApJ, 641, 1060

Maund, J. R., Smartt, S. J., \& Danziger, I. J. 2005, MNRAS, 364, L33

Meynet, G., \& Maeder, A. 2003, A\&A, 404, 975

Misra, K., Pooley, D., Chandra, P., et al. 2007, MNRAS, 381, 280

Müller, E., Fryxell, B., \& Arnett, D. 1991, A\&A, 251, 505

Nadyozhin, D. K. 2003, MNRAS, 346, 97

Rho, J., Jarrett, T. H., Chugai, N. N., \& Chevalier, R. A. 2007, ApJ, 666, 1108 Sahu, D. K., Anupama, G. C., Srividya, S., \& Muneer, S. 2006, MNRAS, 372, 1315

Smartt, S. J., Eldridge, J. J., Crockett, R. M., \& Maund, J. R. 2009, MNRAS, 395,1409

Utrobin, V. P. 2004, Astron. Lett., 30, 293

Utrobin, V. P. 2007, A\&A, 461, 233

Utrobin, V. P., \& Chugai, N. N. 2008, A\&A, 491, 507

Utrobin, V. P., Chugai, N. N., \& Pastorello, A. 2007, A\&A, 475, 973

Vink, J. S., de Koter, A., \& Lamers, H. J. G. L. M. 2001, A\&A, 369, 574

Wang, L., Wheeler, J. C., Höflich, P., et al. 2002, ApJ, 579, 671

Woosley, S. E., \& Heger, A. 2007, Phys. Rep., 442, 269 